\title{
Ron tyrosine kinase receptor synergises with EGFR to confer adverse features in head and neck squamous cell carcinoma
}

\author{
$\mathrm{J} \mathrm{Keller}^{1}$, A S Nimnual ${ }^{1}$, K R Shroyer ${ }^{2}$, C Joy ${ }^{1}$, I Ischenko ${ }^{1}$, C S Chandler ${ }^{1}$, L M Dong ${ }^{3}$, M J Hayman ${ }^{1}$ and \\ E L Chan ${ }^{*} 1,4$ \\ ${ }^{1}$ Department of Microbiology and Molecular Genetics, Stony Brook University, Stony Brook, NY 11794, USA; ${ }^{2}$ Department of \\ Pathology, Stony Brook University, Stony Brook, NY 11794, USA; ${ }^{3}$ Department of Preventive Medicine, Stony Brook University, \\ Stony Brook, NY 11794, USA and ${ }^{4}$ Department of Pediatrics, Division of Hematology/Oncology, HSC, T-11, Room 020, Stony \\ Brook University Medical Center, Stony Brook, NY 11794-8111, USA
}

Background: Although EGFR inhibitors have shown some success in the treatment of head and neck squamous cell carcinomas (HNSCCs), the results are not dramatic. Additional molecular targets are urgently needed. We previously showed that the loss of Ron receptor activity significantly slowed squamous tumour growth and progression in a murine model. Based on these data, we hypothesised that Ron expression confers an aggressive phenotype in HNSCCs.

Methods: We prospectively collected and evaluated 154 snap-frozen, primary HNSCCs for Ron and EGFR expression/ phosphorylation. Biomarker correlation with clinical, pathological and outcome data was performed. The biological responses of HNSCC cell lines to Ron knockdown, its activation and the biochemical interaction between Ron and EGFR were examined.

Results: We discovered that 64.3\% (99 out of 154) HNSCCs expressed Ron. The carcinomas expressed exclusively mature functional Ron, whereas the adjacent nonmalignant epithelium expressed predominantly nonfunctional Ron precursor. There was no significant association between Ron and sex, tumour differentiation, perineural/vascular invasion or staging. However, patients with Ron + HNSCC were significantly older and more likely to have oropharyngeal tumours. Ron + HNSCC also had significantly higher EGFR expression and correlated strongly with phosphorylated EGFR (pEGFR). Newly diagnosed HNSCC with either Ron/ pEGFR or both had lower disease-free survival than those without Ron and pEGFR. Knocking down Ron in SCC9 cells significantly blunted their migratory response to not only the Ron ligand, MSP, but also EGF. Stimulation of Ron in SCC9 cells significantly augmented the growth effect of EGF; the synergistic effect of both growth factors in SCC9 cells was dependent on Ron expression. Activated Ron also interacted with and transactivated EGFR.

Conclusion: Ron synergises with EGFR to confer certain adverse features in HNSCCs.

Ample data over the past 20 years strongly supported an important role of EGFR and its ligands in the development and progression of head and neck squamous cell carcinomas (HNSCCs). Overexpression of EGFR and its ligand, TGF- $\alpha$, had been reported in up to $80 \%$ of HNSCCs (Quon et al, 2001); EGFR mRNA and protein levels were also increased in dysplastic lesions and histologically normal mucosa from HNSCC patients (Ford and Grandis, 2003). In the late 1990s, studies exploring the prognostic value of EGFR expression in HNSCCs began to emerge. A landmark study showed that quantitative difference in TGF- $\alpha$ 
and EGFR protein levels were reliable predictors for adverse outcome in head and neck cancer patients; these biomarkers were superior to the clinical and pathologic factors in predicting clinical outcomes for these patients (Rubin Grandis et al, 1998). Subsequent studies confirmed that high EGFR expression was a robust and independent predictor for survival and local regional relapse (Etienne et al, 1999; Ang et al, 2002). With these data, strategies to block EGFR activity were developed. The agents targeting EGFR moved into clinical trials for HNSCCs in early 2000. Despite the mountain of evidence suggesting the importance of EGFR in HNSCCs, the results of various clinical trials testing different EGFR inhibitors were not as dramatic as one would anticipate. Phase II trials testing the efficacy of various EGFR inhibitors such as gefitinib (Iressa), erlotinib (Tarceva) and cetuximab (Erbitux) in treating recurrent or metastatic HNSCCs only yielded a response rate of $5-10 \%$ with a disease control rate of 40-50\% (Cohen et al, 2003; Soulieres et al, 2004; Baselga et al, 2005). The best result came from a phase III clinical trial that compared radiotherapy alone with radiotherapy plus Erbitux. In the study, Erbitux improved the local regional control and prolonged the progression-free survival of patients with advancedstage HNSCC. However, the overall survival only improved by $10 \%$ and $>50 \%$ of the patients still succumbed to their disease (Bonner et al, 2006). Thus, additional novel therapeutics are needed to improve the efficacy of these inhibitors.

The tyrosine kinase receptor, Ron, is a heterodimeric glycoprotein with disulphide-linked $\alpha$-chain $(35 \mathrm{kDa})$ and $\beta$-chain (150 kDa) (Ronsin et al, 1993; Gaudino et al, 1994). The two chains derive from a single-chain inactive precursor of $185 \mathrm{kDa}$ that undergoes proteolytic cleavage to yield the functional protein. The ligand for Ron is MSP, also known as HGFL (Han et al, 1991; Gaudino et al, 1994; Wang et al, 1995). The structure of Ron is analogous to a family of multifunctional tyrosine kinase receptors that includes c-Met. There is increasing evidence to suggest that Ron expression and/or increased Ron activity is associated with human cancers. Ron was expressed or overexpressed in a variety of primary epithelial tumours (Chen et al, 1997; Maggiora et al, 1998; Willett et al, 1998; Lin et al, 2004; Camp et al, 2005; Cheng et al, 2005; Camp et al, 2007). Increased expression of Ron is also important in tumour formation and growth in experimental animal models (Peace et al, 2001; Chen et al, 2002; Peace et al, 2005; Zinser et al, 2006; Welm et al, 2007). Studies examining the prognostic significance of Ron in cancer patients are beginning to emerge. Ron expression was an independent predictor of distant relapse in a cohort of $103 \mathrm{~T}_{1-2} \mathrm{~N}_{0} \mathrm{M}_{0}$ breast cancer patients; patients with Ron + tumours had a significantly worse 10-year disease-free survival (Lee et al, 2005). Ron expression was also prognostic in gastroesophageal adenocarcinoma (Catenacci et al, 2011) and in a bladder cancer cohort of 183 patients (Cheng et al, 2005). Interestingly, co-expression of Ron and EGFR significantly correlates with tumour recurrence and decreased survival in this bladder cancer cohort (Hsu et al, 2006). The strongest evidence of Ron as a prognostic biomarker came from the analysis of a gene expression data set for the Ron signalling pathway in over 400 breast cancer patients. The coordinate expression of Ron, MSP and MT-SP1 (the protease that activates MSP) independently increased the risk of both metastasis and death in these patients by almost three-fold (Welm et al, 2007). Overall, these translational studies provide early evidence that Ron may be an important biomarker and target for many epithelial cancers.

Using a mouse model of squamous papilloma and carcinoma progression, we discovered that defective Ron signalling resulted in diminished squamous papilloma growth (Chan et al, 2005). Given that EGFR and its ligands play a critical role for tumour growth in this model (Dominey et al, 1993; DiGiovanni et al, 1994; Sibilia et al, 2000; Woodworth et al, 2000; Casanova et al, 2002), and also because of the resemblance of this transgenic mouse model to the progression of HNSCCs (Lu et al, 2006), we hypothesise that Ron has a complementary role to EGFR in HNSCCs. In this study, we examined the role of Ron and its relationship to EGFR in HNSCC patients and cell lines. We found that Ron synergises with EGFR to confer certain adverse features in head and neck cancers.

\section{MATERIALS AND METHODS}

Collection of primary HNSCCs and the associated clinical data. Snap-frozen primary HNSCCs were collected prospectively for this study through the Cooperative Human Tissue Network (CHTN) from 2006 to 2010. When HNSCC patients presented to the CHTN institutions for surgical evaluation, informed consent for participation in tumour banking and research was obtained by the CHTN staff at the local institutions. After the patients consented, the accrued tumours were sent to our laboratory in dry ice within $24 \mathrm{~h}$ after surgical resection. In addition, 1 haematoxylin and eosin (H\&E)-stained and 10 unstained slides from each specimen were received at the same time. At the time of procurement, the CHTN staff also reviewed medical charts of patients to obtain relevant clinical information such as age, sex, race, prior treatment, imaging reports, history of smoking and alcohol use. Pathology reports including detailed information such as tumour location, nodal involvement, size and number of involved nodes, histological features and staging were sent to us deidentified at the same time. A sample of these clinical data is shown in Supplementary Figures S3-S5. To avoid investigator bias, Ron and EGFR analyses were performed before follow-up data were obtained. Follow-up information included the treatment after surgery, follow-up notes, imaging reports if available and the time of follow-up from initial surgery. See Supplementary Figure S6 for a sample of the follow-up information. Exempted IRB approval for this study was obtained at our institution.

Cell lines, reagents and antibodies. We obtained the following HNSCC cell lines from the American Type Culture Collection (Manassas, VA, USA): SCC9, SCC15 and CAL27. MDA 1386 was kindly provided by Dr Kepal Patel from New York University. The COS1 and 3T3 Ron cells were previously obtained and generated (Ischenko et al, 2003; Agazie and Hayman, 2003b). The American Type Culture Collection routinely performs cell line characterisation by STR profiling and the HNSCC cell lines were passaged $<6$ months after resurrection. The SCC9, SCC15 and MDA 1386 cells were grown in 1:1 mixture DMEM/Ham F12 and RPMI (Gibco, Grand Island, NY, USA) respectively, whereas CAL27, COS1 and 3T3 Ron cells were maintained in DMEM. All media were supplemented with $10 \%$ FBS, $1 \%$ penicillin/streptomycin (Gibco) at $37^{\circ} \mathrm{C}$ in a $5 \% \mathrm{CO}_{2}$-humidified incubator. The following antibodies were used: for immunoprecipitation: cetuximab (ImClone, New York, NY, USA), EGFR rabbit antibody (Hayman et al, 1986), human MSPR antibody (R\&D Systems, Minneapolis, MN, USA), Met antibody (Santa Cruz Biotechnology, Santa Cruz, CA, USA), mouse, rabbit and human IgG (Bethyl Lab, Montgomery, TX, USA); for western blotting: anti-phosphotyrosine 4G10 antibody (Millipore, Temecula, CA, USA), Ron C20 antibody (Santa Cruz Biotechnology), EGFR rat antibody (Agazie and Hayman, 2003b), Met, MAPK and phospho-MAPK antibodies (Cell Signaling Technology, Danvers, MA, USA); for immunohistochemistry: Ron C20 and EGFR antibodies (Cell Signaling Technology). The fluorescent labelled secondary anti-rabbit, mouse and rat antibodies were purchased from Molecular Probes (Grand Island, NY, USA). The following growth factors were used in the migration and XTT assays: EGF (Gibco) and MSP (R\&D Systems). A retroviral shRNA vector directed against Ron was kindly provided by Dr J Freeman (Zhao et al, 2008). 
Processing of primary HNSCCs for protein analyses by immunoprecipitation followed by western blot (IPW) and immunohistochemistry (IHC). Primary HNSCCs were homogenised in $1 \mathrm{ml}$ of Triton X lysis buffer ( $50 \mathrm{~mm}$ Tris-Cl, $\mathrm{pH}=8$, $150 \mathrm{~mm} \mathrm{NaCl}$ and $1 \%$ Triton X-100) containing protease inhibitor and activated sodium orthovanadate using the POLYTRON system PT 10-35GT (Kinematica AG, Bohemia, NY, USA). The homogenates were spun down; pellets were discarded and the supernatants were saved for protein analyses. Protein concentrations were determined by Bradford assay (Thermo Scientific, Rockford, IL, USA).

Along with the Ron/EGFR antibody, $1 \mathrm{mg}$ aliquot of tumour lysate was incubated overnight. The antibody-protein conjugates were extracted using protein G Sepharose beads and the protein was eluted by incubation at $95^{\circ} \mathrm{C}$ in loading buffer. The proteins were subsequently resolved by $8 \%$ polyacrylamide gel electrophoresis under reducing conditions and transferred to Immobilon-P membrane (Millipore). Western blotting was performed first with the 4 G10 antibody and then with Ron/EGFR antibody. Fluorescent secondary antibodies were used to develop the western blots so that Ron/EGFR expression and phosphorylation could be quantified by the Odyssey Infrared Imaging System (LI-COR Biosciences, Lincoln, NE, USA).

Unstained HNSCC sections were deparaffinised, rehydrated and antigen retrieval was performed by microwave heating in sodium citric solution. After washing with PBS, the sections were treated with blocking serum (Vectastain ABC-AP kit, Vector Laboratories, Burlingame, CA, USA) and incubated overnight in $4{ }^{\circ} \mathrm{C}$ with Ron/ EGFR antibody at $1: 100 / 1: 50$ dilution, respectively. The tumour sections were subsequently washed with PBS and stained using the $\mathrm{ABC}-\mathrm{AP}$ kit and the Vector Red alkaline phosphatase substrate solution. The H\&E-stained HNSCC sections were reviewed by a surgical pathologist (KRS) to confirm the diagnosis and determine tumour viability as well as the ratio of tumour to stroma surface area. Ron IHC was also graded by the pathologist (KRS). A subjective scale was defined and used to score the overall impression of Ron IHC stain in the carcinoma: $0=$ no staining, $1+=$ weak staining and $2+=$ moderate to strong staining. In addition, the proportion of Ron + cancer cells in the representative tumour section was estimated on a scale from $0 \%$ to $100 \%$ for each staining level. The total IHC score was defined as $\%$ Ron + cells $\times$ degree of staining. Histological assessment was performed without knowledge of the molecular testing results and clinical data.

Correlation and survival analyses. To examine the association between Ron expression and tumour characteristics, the following inclusion and exclusion criteria were established: (1) only histologically confirmed HNSCCs of the primary sites were included; (2) both newly diagnosed and recurrent HNSCCs were included; (3) patients with recurrent HNSCCs who were previously treated with surgical resection alone were included; (4) HNSCCs with viable tumour to stroma ratio of $\leqslant 50 \%$ were excluded to avoid false negative biochemical results; and (5) HNSCC patients who had been treated with radiation and/or chemotherapy before tumour banking were excluded.

For the survival analysis, we included both untreated and previously treated patients. The inclusion and exclusion criteria for this analysis were: (1) histologically confirmed newly diagnosed and recurrent HNSCCs were included; (2) both previously treated and untreated HNSCC patients were included; (3) cases with no or unclear follow-up information were excluded; (4) cases from CHTN institutions that do not have IRB approval to obtain followup information were excluded; and (5) HNSCCs with viable tumour to stroma ratio of $\leqslant 50 \%$ were excluded.

Generation of SCC9/shRNA-Ron stable clones. Retroviral vector expressing shRNA against human Ron was kindly provided by Dr J Freeman (Zhao et al, 2008). Phoenix E cells were transfected with the shRNA-Ron retroviral vector or control vector with scrambled shRNA sequence. Virus-containing supernatant from these cells was added to SCC9 cells for $6 \mathrm{~h}$. Stable clones were selected with $2 \mu \mathrm{g} \mathrm{ml}^{-1}$ puromycin and Ron knockdown was confirmed by western blot.

Migration and XTT assay. Migration assay was performed as previously described (Feres et al, 2009). Briefly, SCC9 cells were grown in $1 \%$ FBS media overnight; $10^{5}$ cells were added to the top well of a 24 -well, $0.8 \mu \mathrm{m}$ pore Transwell filter insert (Costar, Corning, NY, USA). Media added to the bottom well contained $10 \mathrm{ng} \mathrm{ml}^{-1} \mathrm{EGF}$ or $100 \mathrm{ng} \mathrm{ml}^{-1} \mathrm{MSP}$. After $6 \mathrm{~h}$, cells that remained in the top chamber were removed with a cotton swab, the migrated cells were fixed and DNA was labelled with Hoechst. The number of migrating cells per high-power field across the field of view was counted on a fluorescent microscope under $\times 20$ magnification and averaged.

The XTT proliferation assay was performed as per the manufacturer's recommendation (ATCC, Manassas, VA, USA). Briefly, CAL27 or SCC9 cells were seeded at $7.5 \times 10^{4}$ cells per well in a 96-well plate in triplicates. The cells were grown in $0.1 \%$ FBS media for 2 days and then in media containing $50 \mathrm{ng} \mathrm{ml}^{-1}$ EGF or $100 \mathrm{ng} \mathrm{ml}^{-1} \mathrm{MSP}$ or both for 1 day. Activated-XTT reagent was prepared and added to the cells the following day as per protocol. Cell proliferation was determined at 30-min time interval after the addition of XTT reagent by a spectrophotometer, which measured absorbance at 465 and $660 \mathrm{~nm}$. The specific absorbance of each sample was calculated as follows: $\mathrm{A}_{475 \mathrm{~nm}}$ (test)- $\mathrm{A}_{475 \mathrm{~nm}}$ (blank)$\mathrm{A}_{660 \mathrm{~nm}}$ (test). The migration and XTT experiments were performed at least three separate times for each cell line.

Biochemical analysis. The COS1 cells were transiently transfected with a previously obtained Ron expression construct (Ischenko et al, 2003) or its corresponding control vector (PClneo) using Fugene 6 as per the manufacturer's protocol (Promega Corporation, Madison, WI, USA). Phosphorylation of EGFR was determined by IPW as described. The CAL27 cells were serum starved overnight and stimulated with $10 \mathrm{ng} \mathrm{ml}^{-1} \mathrm{EGF}$ or $100 \mathrm{ng} \mathrm{ml}^{-1} \mathrm{MSP}$ for $20 \mathrm{~min}$ the next day. Ron, EGFR and Met IPW were performed on the cell lysates. The cell lysates were also analysed for MAPK and RTK signalling. A phospho-RTK array was used and developed as per the manufacturer's protocol (R\&D Systems). Each biochemical experiment was repeated to confirm the findings.

Statistical analysis. Statistical analyses were performed using SPSS Statistics 16.0 (SPSS Inc., Chicago, IL, USA) and R (version 2.15.2; Vienna, Austria). The $\chi^{2}$ test was used to examine the association between Ron and tumour characteristics measured in discrete variables. For the continuous variables such as age, smoking (pack years), tumour size (log volume) and Ron/EGFR expression ( $\log$ value), comparisons between Ron + and Ron - as well as pEGFR + and pEGFR - group were performed using two samples $t$-test. Ron/EGFR expression and tumour size data were log transformed to normalise the distributions for comparison. Pearson's correlation coefficient was determined and used to describe the relationship between Ron and EGFR expression level. Level of statistical significance is $5 \%$. When multiple comparisons were performed, the Bonferroni adjusted $P$-value was used to determine the level of significance. In the survival analysis, an event was defined as relapse or progression of the head and neck cancer after the tumour banking surgery and time to event was months from the date of tumour banking surgery until the date of an event or the last follow-up. Event-free survival (EFS) curves were constructed using the Kaplan-Meier method and compared using log-rank test. The Cox proportional hazards model was used to assess the effect of Ron or the combination of Ron and pEGFR expression in the presence of overall stage (1/2 vs 3/4) and prior treatment (chemotherapy/radiation or not), the two clinically 


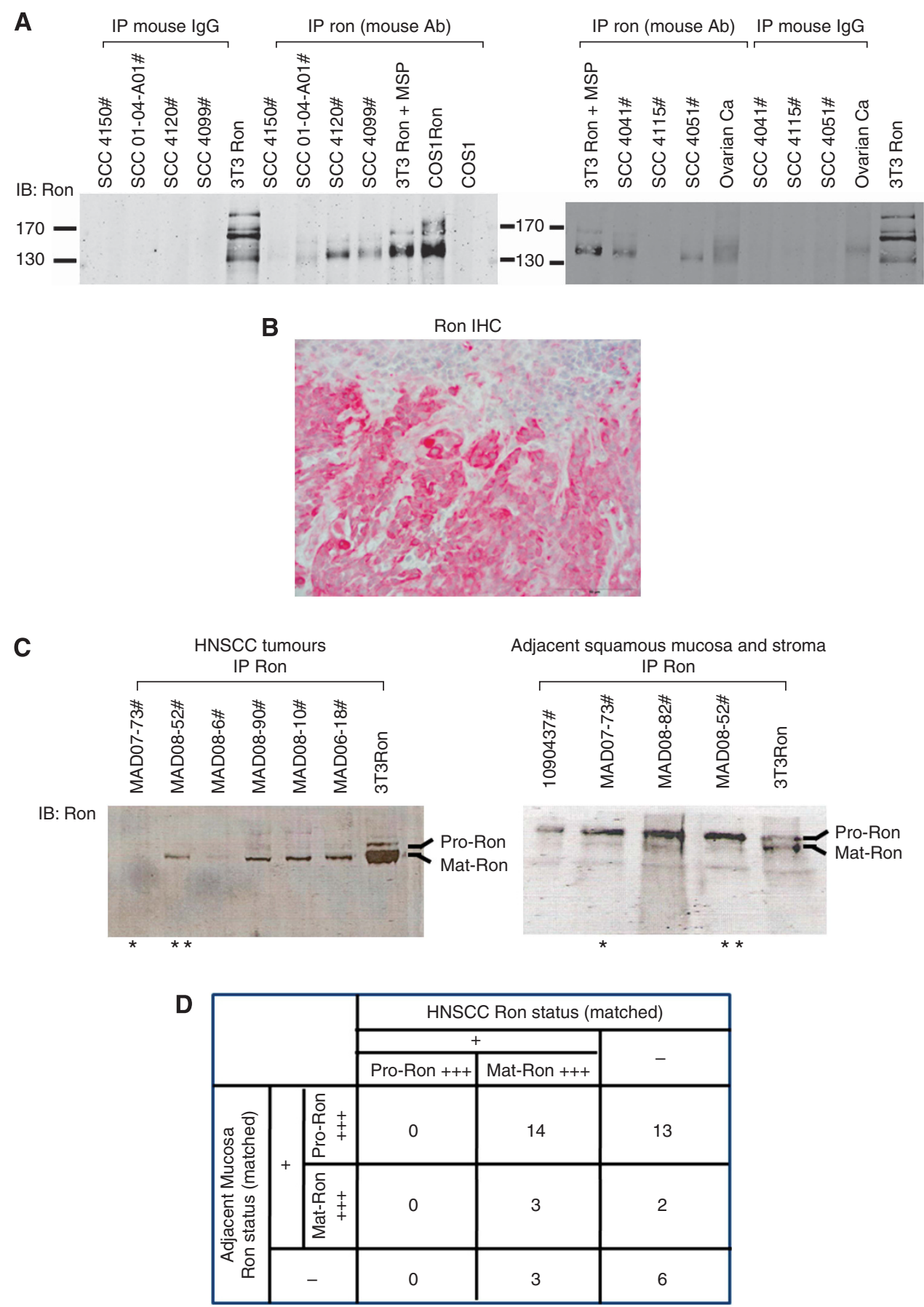

Figure 1. Ron status in HNSCCs. (A) A representative IPW analysis of Ron expression in primary HNSCCs. Control immunoprecipitations with normal mouse IgG were negative; COS1 or 3T3 cells overexpressing Ron (COS1Ron or 3T3Ron) were positive controls. (B) Ron immunohistochemistry of a representative HNSCC ( $\times 100)$. Note that Ron was localised primarily in the squamous carcinoma tumour cells and only minimal staining was detected in the tumour stroma. (C) A representative IPW analysis of Ron expression in primary HNSCCs and adjacent squamous mucosa and stroma. Two of the samples were matched as indicated by the asterisks. Pro-Ron is the precursor/unprocessed form of Ron at $170 \mathrm{kDa}$ ( $\alpha$ - and $\beta$-chains), whereas Mat-Ron is the functional/processed form of Ron at $150 \mathrm{kDa}$ ( $\beta$ chain). (D) Summary of Ron status in the paired HNSCCs and matched adjacent squamous mucosa and stroma ( $n=41$ pairs of matched samples).

relevant and potentially prognostic factors in our patient population.

\section{RESULTS}

Ron expressed in a high percentage of primary HNSCCs. Ron and EGFR phosphorylation/expression status was determined by IPW in 154 primary HNSCCs. Although IHC using the C20 Ron antibody has been the method of choice to determine Ron expression in primary tumours for multiple translational studies (Cheng et al, 2005; Lee et al, 2005; Hsu et al, 2006; Catenacci et al, 2011), we discovered that this antibody gives significant background signals on tumour lysates. As shown in Supplementary Figure S1, tumour lysate from bladder cancer known to express Ron (Hsu et al, 2006) exhibited multiple bands on western blot from 55 to $130 \mathrm{kDa}$. This crossreactivity could potentially yield 
Table 1. Comparison of clinicopathologic features between Ron + and Ron - HNSCCs

\begin{tabular}{|c|c|c|c|}
\hline & $\begin{array}{c}\text { Ron }+ \text { HNSCCs, } \\
n=83(65.4 \%)\end{array}$ & $\begin{array}{c}\text { Ron - HNSCCs, } \\
n=44(34.6 \%)\end{array}$ & $\boldsymbol{P}$-value \\
\hline Age (years) & $61.1 \pm 11.8$ & $55.7 \pm 9.4$ & $<0.01$ \\
\hline \multicolumn{4}{|l|}{ Sex } \\
\hline $\begin{array}{l}\text { Female } \\
\text { Male }\end{array}$ & $\begin{array}{l}18(21.7 \%) \\
65(78.3 \%)\end{array}$ & $\begin{array}{l}10(22.7 \%) \\
34(77.3 \%)\end{array}$ & NS \\
\hline \multicolumn{4}{|l|}{ Tumour sites } \\
\hline $\begin{array}{l}\text { Oropharynx } \\
\text { Larynx }\end{array}$ & $\begin{array}{l}68(81.9 \%) \\
15(18.1 \%)\end{array}$ & $\begin{array}{l}29(65.9 \%) \\
15(34.1 \%)\end{array}$ & 0.05 \\
\hline $\begin{array}{l}\text { Smoking (pack } \\
\text { years) }\end{array}$ & $37.8 \pm 36.2$ & $36.2 \pm 29.7$ & NS \\
\hline
\end{tabular}

Differentiation

\begin{tabular}{|l|c|r|l|}
\hline Missing & $2(2.4 \%)$ & 0 & \\
Well & $12(14.5 \%)$ & $6(13.6 \%)$ & \\
Moderate & $46(55.4 \%)$ & $31(70.5 \%)$ & \\
Poor & $23(27.7 \%)$ & $7(15.9 \%)$ & NS \\
\hline
\end{tabular}

Perineural invasion

\begin{tabular}{|l|l|l|l|}
\hline Missing & $22(26.5 \%)$ & $14(31.8 \%)$ & \\
Yes & $35(42.2 \%)$ & $18(40.9 \%)$ & \\
No & $26(31.3 \%)$ & $12(27.3 \%)$ & NS \\
\hline
\end{tabular}

Lymphovascular invasion

\begin{tabular}{|l|c|c|c|}
\hline Missing & $15(18.1 \%)$ & $2(4.5 \%)$ & \\
Yes & $23(27.7 \%)$ & $19(43.2 \%)$ & \\
No & $45(54.2 \%)$ & $23(52.3 \%)$ & NS \\
\hline Tumour size & $3.23 \pm 1.25$ & $2.97 \pm 1.35$ & NS \\
$\left(\log \mathrm{cm}^{3}\right)$ & & & \\
\hline
\end{tabular}

\begin{tabular}{|l|c|c|c|}
\hline \multicolumn{5}{|l|}{ T stage } & $1(1.2 \%)$ & $1(2.3 \%)$ & \\
\hline Tx & $23(27.7 \%)$ & $15(34.1 \%)$ & \multirow{2}{*}{ NS } \\
Tis/T1/T2 & $59(71.1 \%)$ & $28(63.6 \%)$ & \\
T3/T4 & \multicolumn{3}{|l|}{} \\
\hline N stage & $11(13.2 \%)$ & $5(11.4 \%)$ & \\
\hline Nx & $40(48.2 \%)$ & $22(50 \%)$ & NS \\
NO/N1 & $32(38.6 \%)$ & $17(38.6 \%)$ & \\
N2/N3 & $9(10.8 \%)$ & $5(11.4 \%)$ & \\
\hline Overall stage & $9(10.8 \%)$ & $5(11.4 \%)$ & NS \\
\hline Missing & $65(78.4 \%)$ & $34(77.2 \%)$ & \\
1-2 & $7(8.4 \%)$ & $4(9.1 \%)$ & \\
3-4 & & & \\
\hline Recurrence & &
\end{tabular}

Abbreviations: $\mathrm{HNSCC}=$ head and neck squamous cell carcinoma; NS = not significant.

false positive results in IHC, thereby reducing the specificity of the test. To overcome this flaw, we used a highly specific mouse Ron antibody to first immune precipitate the receptor from the tumour lysates. Then, a second Ron antibody, C20, was used to detect the protein and determine its molecular weight on western blot. With this approach, the crossreactivity was eliminated and Ron expression was identified with high specificity (Supplementary Figure S1). We compared this IPW method of detecting Ron with Ron IHC. As shown in Supplementary Figure S2, there are large number of both Ron-positive and Ron-negative IPW cases in the low range of Ron IHC score (0-50). Assuming that Ron IPW result is the gold standard, Ron IHC using the C20 Ron antibody not only gives false positive results, but also false negatives. Using a Ron IHC score of 35 as the cutoff point, the percentages of false positive and false negative IHC were roughly $44 \%$ and $36 \%$, respectively. These data argue to identify a better Ron antibody than C20 for IHC use and translational studies.

Using IPW as the method of Ron detection, we found that primary HNSCCs expressed various levels of Ron (Figure 1A). Some expressed levels of Ron equivalent to Ron overexpression driven in 3T3/COS1 cells by an expression vector (i.e., SCC4120\#), whereas others expressed lower to undetectable levels (i.e., SCC4115\# or SCC01-04-A01\#). Similar to our previous finding (Keller et al, 2010), only 58.4\% (90 out of 154) HNSCCs expressed phosphorylated EGFR (pEGFR) as detected by IPW. Although the IPW method of detecting Ron is very specific, the shortcoming is its inability to localise Ron expression to a specific cell type. This is because of the heterogeneity of the homogenised tumour lysates. Thus, IHC was performed to confirm Ron expression in the cancer cells. As shown in Figure 1B, Ron was expressed primarily in the malignant squamous epithelial cells, but was not detected in stromal cells. Ron expression in the nonmalignant squamous mucosa adjacent to the tumour also localised mainly to the squamous epithelium. This was confirmed in all HNSCC sections tested. By using a combination of IPW and IHC, we could definitively conclude that of the 154 HNSCCs analysed, 64.3\% (99 out of 154) expressed Ron.

HNSCCs predominantly expressed functional Ron. Of the 154 HNSCC cases, 41 had adjacent noncancerous squamous epithelium and stroma from the same patients. Ron status was determined by IPW in these matched tissues. Interestingly, the precursor form of Ron (Pro-Ron) was detected in the majority (27 out of 41) of the matched noncancerous tissues, but Pro-Ron was not the predominant form in any of the corresponding Ron + HNSCCs (Figure 1C and D). Ron is first translated into a single-chain polypeptide (Pro-Ron) that has to undergo proteolytic cleavage to yield the mature, functional cell surface protein (Mat-Ron). Thus, the Pro-Ron seen in the noncancerous tissues is not likely to be functional. Interestingly, a small percentage (5 out of 41) of the matched noncancerous tissues expressed mature Ron; in two of these five samples, the adjacent HNSCCs did not express Ron (Figure 1D). In addition, even when the matched noncancerous tissues expressed Pro-Ron, close to 48\% (13 out of 27) of the adjacent head and neck tumours did not express Ron. Overall, these data suggested that the processing of Ron to the mature functional form played a role in HNSCC progression.

Ron expression in HNSCCs did not correlate with staging, but strongly associated with EGFR expression and pEGFR. After Ron, EGFR and pEGFR expression were known in these HNSCCs, we performed analyses to determine if Ron expression is associated with certain disease characteristics. The cohort of HNSCCs composed of patients who had not been previously treated with radiation and/or chemotherapy $(n=127)$ and those who had received radiation and/or chemotherapy before surgical resection for tumour banking $(n=27)$. We defined these two patient populations as untreated and previously treated patient cohorts. The clinical and pathological characteristics of these two cohorts are summarised in Supplementary Tables S1 and S2. The percent distributions of all characteristics were very similar between the two cohorts except that more recurrent tumours were seen in the previously treated patient cohort $(51.9 \%$ vs $8.7 \%)$. This finding was not unexpected.

Next, we examined the association between Ron expression and multiple clinical, pathological and molecular features in the untreated patient cohort. Although no significant association between Ron expression and sex, tumour differentiation, presence of perineural/vascular invasion, tumour size or staging was 
A

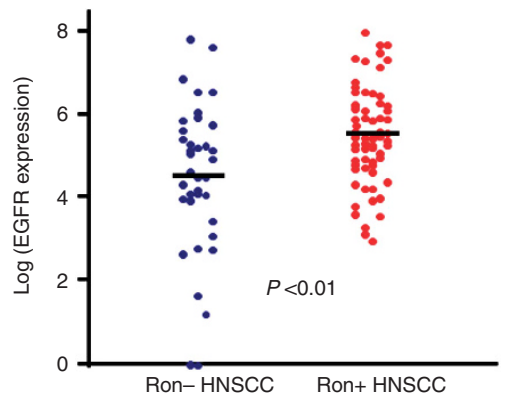

C

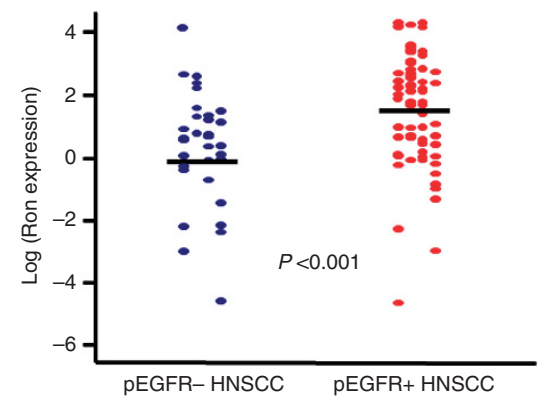

E

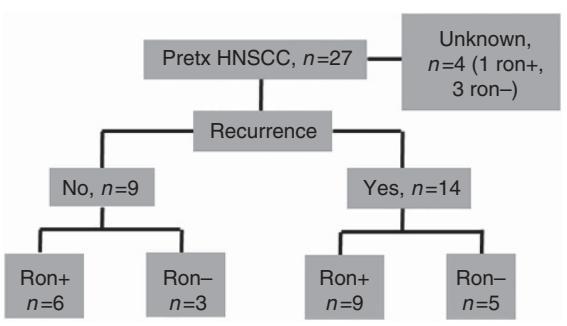

B

\begin{tabular}{|l|c|c|}
\hline & Ron- & Ron+ \\
\hline pEGFR+ & 15 & 62 \\
\hline pEGFR- & 29 & 21 \\
\hline
\end{tabular}

$P<0.001$
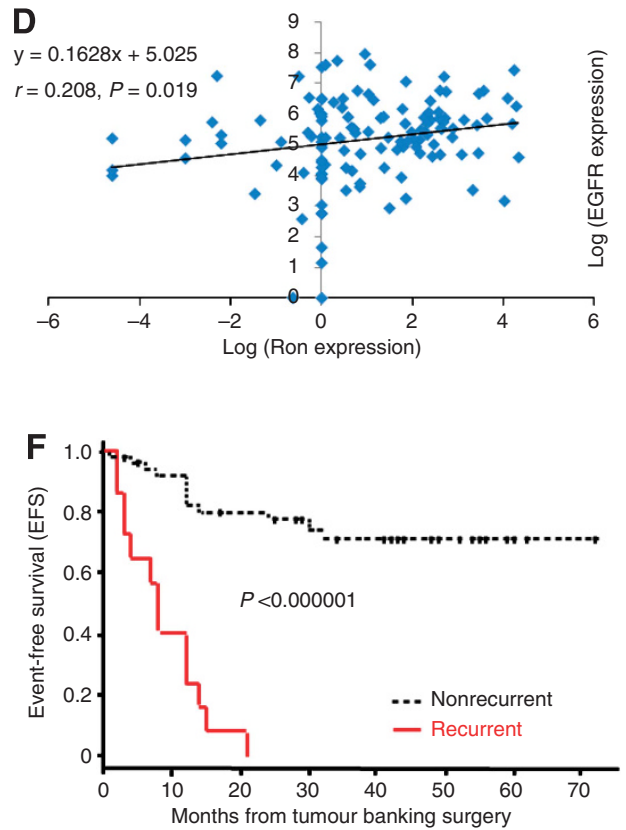

G

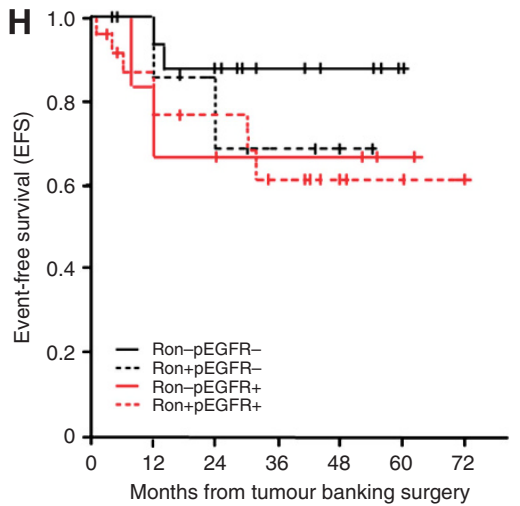

Figure 2. Correlation analysis. (A) Comparison of EGFR expression between Ron $+(n=83)$ and Ron $-(n=44)$ HNSCCs. The expression of EGFR was normalised on a logarithmic scale. (B) Association between Ron expression and phosphorylated EGFR in the same tumour. (C) Comparison of Ron expression between pEGFR + $(n=77)$ and pEGFR - $(n=50)$ HNSCCs. Ron expression was normalised on a logarithmic scale. (D) Scattergram of Ron and EGFR expression level. The best fit line was drawn and calculated as shown with Pearson's correlation coefficient, $r=0.208$. (E) Flow diagram of the recurrent cases with Ron expression in the previously treated patient cohort. Pretx=previously treated. (F) Kaplan-Meier plot comparing event-free survival between recurrent and nonrecurrent HNSCCs $(n=72)$. (G) Kaplan-Meier plots comparing event-free survival between recurrent Ron + and Ron - HNSCCs (left panel) as well as recurrent pEGFR + and pEGFR - HNSCCs (right panel), $(n=15)$. (H) Kaplan-Meier plot comparing event-free survival between nonrecurrent Ron - pEGFR - , Ron $-p E G F R+$, Ron + pEGFR - and Ron + pEGFR + HNSCCs $(n=56)$.

detected, patients with Ron + HNSCCs were significantly older (Table 1). In addition, a significantly higher percentage of Ron + HNSCCs was located in the oropharynx (Table 1). Ron + HNSCCs also had significantly higher EGFR expression and this correlated strongly with the EGFR being active as judged by tyrosine phosphorylation, pEGFR, in the same tumours (Figure 2A and B). Similarly, pEGFR + HNSCCs had significantly higher Ron expression (Figure 2C). In addition, there is a strong association between Ron and EGFR expression level. As shown in Figure 2D, a significant linear correlation between EGFR and Ron expression 


\begin{tabular}{|c|c|c|c|}
\hline & No. of cases & RR $(95 \% \mathrm{Cl})$ & $P$-value \\
\hline \multicolumn{4}{|c|}{ Ron and pEGFR status } \\
\hline $\begin{array}{l}\text { Ron + pEGFR + } \\
\text { Ron - pEGFR + } \\
\text { Ron + pEGFR - } \\
\text { Ron - pEGFR - }\end{array}$ & $\begin{array}{r}24 \\
8 \\
8 \\
16\end{array}$ & $\begin{array}{c}2.42(0.47-12.5) \\
2.71(0.38-19.3) \\
2.76(0.37-20.5) \\
1\end{array}$ & $\begin{array}{c}0.29 \\
0.32 \\
0.32 \\
- \\
\end{array}$ \\
\hline \multicolumn{4}{|l|}{ Staging } \\
\hline $\begin{array}{l}1 / 2 \\
3 / 4 \\
\text { Missing }\end{array}$ & $\begin{array}{r}8 \\
45 \\
3\end{array}$ & $\begin{array}{c}1 \\
1.60(0.18-14.4) \\
-\end{array}$ & $\overline{0.67}$ \\
\hline \multicolumn{4}{|c|}{ Treatment (Rad/Chemo) } \\
\hline $\begin{array}{l}\text { Yes } \\
\text { No } \\
\text { Missing }\end{array}$ & $\begin{array}{r}37 \\
13 \\
6\end{array}$ & $\begin{array}{c}2.87(0.32-26.0) \\
1 \\
-\end{array}$ & $\begin{array}{c}0.35 \\
-\end{array}$ \\
\hline $\begin{array}{l}\text { Abbreviations: Cher } \\
\text { squamous cell car } \\
\text { Rad=radiation; RR }\end{array}$ & $\begin{array}{l}\text { emotherapy; } \mathrm{Cl}= \\
\text { pEGFR = phosph } \\
\text { e risk. }\end{array}$ & $\begin{array}{l}\text { dence interval; HNS } \\
\text { ted epidermal gro }\end{array}$ & $\begin{array}{l}\text { head and } \\
\text { actor rece }\end{array}$ \\
\hline
\end{tabular}

in the tumours was detected (Pearson's correlation coefficient, $r=0.208, \quad P=0.019)$. Recurrent HNSCCs, whether or not previously treated with chemotherapy and/or radiation, were however no more likely to express Ron or pEGFR (Table 1 and Figure 2E).

HNSCCs that expressed either Ron/pEGFR or both have an inferior EFS than those without Ron and pEGFR expression. We then performed survival analysis to determine if Ron is a prognostic biomarker. Because of an IRB constraint at two of the CHTN institutions, there was a lack of follow-up data in more than half the cases $(n=82)$. Thus, only a total of 72 cases were eligible for the outcome analysis. The median follow-up time for these patients was 22 months. The initial exploration revealed that the single most dominant and significant independent prognostic factor for EFS was recurrence (Figure 2F; $P<0.000001$, log-rank test). This finding was confirmed by Cox regression analysis with adjustment for Ron, pEGFR, stage and treatment $(R R=10.7$, $P<0.000001)$. This was not unexpected because tumours that returned after initial treatment were by nature highly aggressive and therefore had a high tendency to progress or recur again. However, closer examinations indicated that the effect of Ron or pEGFR among recurrent HNSCCs was different than its effect among patients with nonrecurrent tumours. In fact, for those patients with recurrent HNSCCs $(n=15)$, all progressed/recurred within 2 years from surgery regardless of their Ron and/or pEGFR status (Figure $2 \mathrm{G}$ ). This implies that Ron and pEGFR might not have an effect on the outcome of the recurrent HNSCCs. Thus, we conducted the main survival analyses on patients with newly diagnosed, nonrecurrent HNSCCs $(n=56)$. One case with unknown recurrent status was excluded. Among these 56 patients, those with Ron or pEGFR, or both, tend to have lower EFS than those without ( $\sim 20 \%$ difference; Figure $2 \mathrm{H})$. Although the effect of Ron on EFS did not reach statistical significance, pEGFR and staging also did not reach statistical significance as independent predictors for worse EFS in this patient population, even though both are known prognostic factors (Table 2). The lack of statistical significance is most likely because of a small sample size as suggested by the wide $95 \%$ confidence interval. Nevertheless, the relative risk of lower EFS for Ron + pEGFR - tumour $(R R=2.76)$ was very similar to that of Ron $-\mathrm{pEGFR}+$ tumour $(\mathrm{RR}=2.71)$.
This suggested that the prognostic effect of Ron and pEGFR might be similar. Consistent with this suggestion, HNSCCs with both Ron and pEGFR expression had the lowest EFS among all groups (Figure $2 \mathrm{H}$ ). Overall, these outcome data suggested that Ron expression, especially in combination with pEGFR, may impart a lower EFS for newly diagnosed HNSCC patients.

Ron synergises with EGFR to confer migratory and growth advantages to HNSCC cells. To determine the biological function of Ron in HNSCCs, we turned to an analysis of HNSCC cell lines. We first screened HNSCC cell lines for Ron and EGFR expression. As expected, all of the cell lines expressed EGFR (Figure 3B). Endogenous Ron expression varied among the different cell lines with higher levels detected in CAL27 and SCC9 than MDA1386 and SCC15 (Figure 3A). Next, stable SCC9 clones with Ron knockdown were generated using shRNA against Ron and a vector control. Reduction of Ron expression by $\sim 80 \%$ was confirmed by western blot; the knockdown had no effect on EGFR expression (Figure 3C). Migration assays were performed using these stable clones. As shown, the downregulation of Ron not only reduced the migration of SCC9 cells in response to MSP, but also reduced the baseline cell migration and migration in response to EGF (Figure 3D), even though the cells expressed equivalent levels of EGFR (Figure 3C). This result suggested that Ron expression per se has functional consequences for EGF signalling. Then, we performed XTT proliferation assay to examine if stimulation of Ron augments the effect of EGF on cell growth. The growth rate of SCC9 cells after stimulation with EGF or MSP alone was not significantly increased compared with unstimulated cells; on the other hand, the simultaneous addition of both growth factors significantly increased the growth rate above the baseline (Figure 3E). Similar trend of this synergism was observed in CAL27 cells as well (Supplementary Figure S7). To confirm that this effect was Ron dependent, we performed similar XTT assays on the SCC9 clone with knockdown of Ron. Interestingly, not only was the synergistic growth effect of MSP and EGF blunted in these cells, their response to EGF was also inhibited (Figure $3 \mathrm{~F}$ ). This result is consistent with what was observed in the migration assays (Figure 3D). Overall, the data suggested a synergistic biological effect between Ron and EGFR on HNSCC cell growth.

Activated Ron biochemically interacts with EGFR. To investigate potential biochemical interaction between Ron and EGFR, we established an overexpression cell system for our initial analyses. Transient overexpression of Ron in COS1 cells led to transactivation of EGFR (Figure 4A). This result is consistent with other overexpression cell systems that examined Ron/EGFR interaction (Peace et al, 2003). As overexpressing Ron might artificially force its interaction with EGFR, we performed similar biochemical analysis by activating endogenous Ron in CAL27 cells. Consistent with the above result, activating Ron by MSP in CAL27 cells transphosphorylated endogenous EGFR (Figure 4B, upper panel). As Met was baseline activated in these CAL27 cells (Figure $4 \mathrm{C}$ and Supplementary Figure S8, upper panel), we confirmed that the EGFR antibody did not pull down phosphorylated Met/Ron to account for the phosphorylated bands seen on the western blot (Supplementary Figure S8). To ensure that Ron is activated by MSP, we examined MAPK signalling post MSP as a sensitive readout of Ron activation. As expected, MAPK was phosphorylated post MSP stimulation (Figure $4 \mathrm{~B}$, lower panel). Using the R\&D phosphoarray, we also detected Ron phosphorylation post EGF stimulation (Figure 4C). This showed that EGFR activation transphosphorylated Ron. Interestingly, EGFR activation by EGF also transphosphorylated other RTKs, including not only the known heterodimers, ErbB2 and 4 (Olayioye et al, 2000), but also Mer2, Tie-2, RYK, DDR1 and ROR2, which have not been shown to interact with EGFR. Lastly, activated Ron 

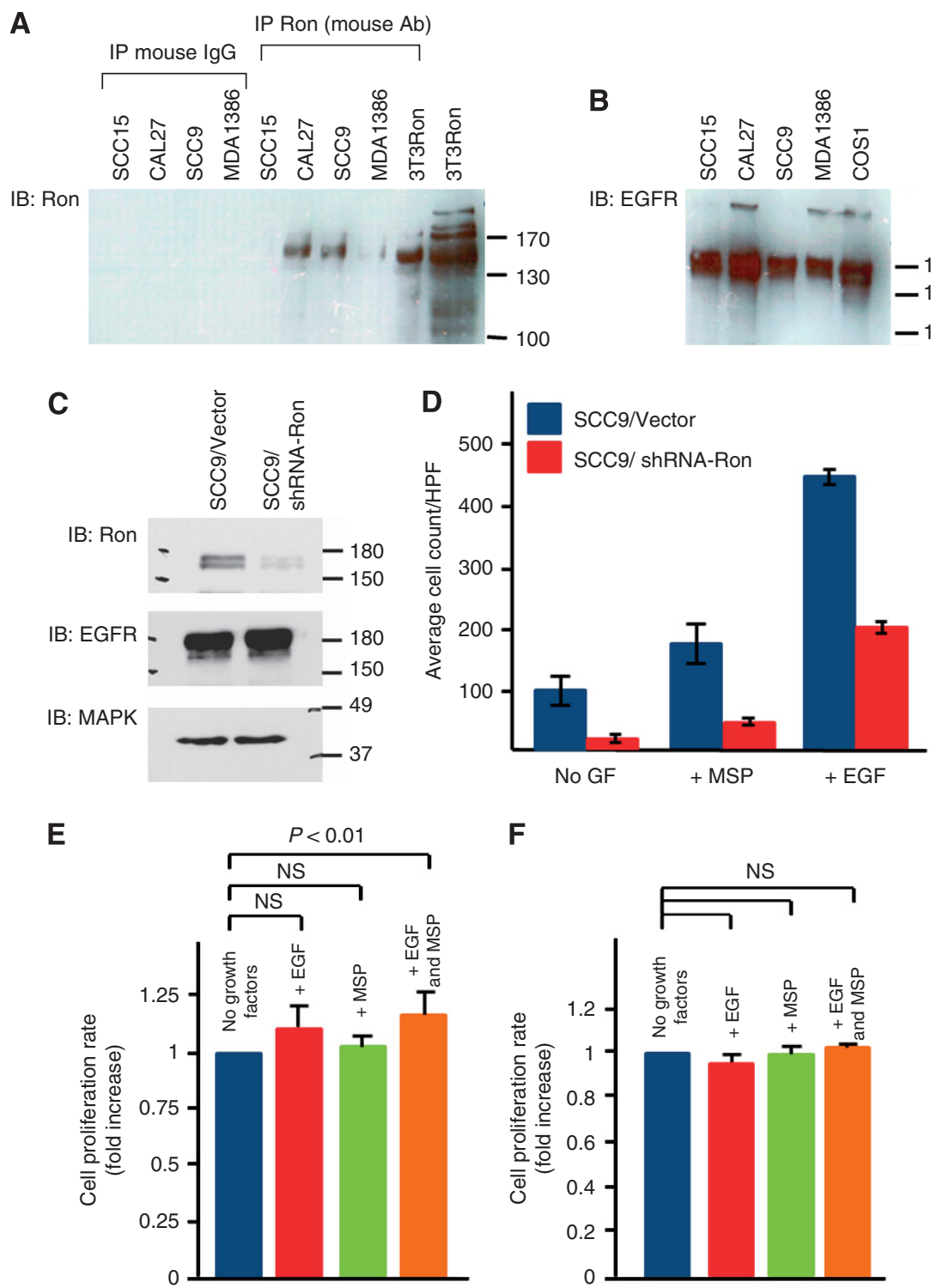

Figure 3. Functional assays of Ron + HNSCC cells. (A) Ron expression in a panel of HNSCC cell lines. The 3T3Ron was positive control. Control precipitations with normal mouse lgG were all negative. (B) The expression of EGFR in a panel of HNSCC cell lines. The COS1 cells known to express EGFR (Agazie and Hayman, 2003a) were the positive control. (C) Ron expression of SCC9 cells stably expressed shRNA against Ron (SCC9/shRNA-Ron) or scrambled sequences (SCC9/Vector). The blot was probed successively with antibodies against Ron, EGFR and finally MAPK as a loading control. (D) Migration of SCC9/shRNA-Ron and SCC9/Vector cells in response to EGF or MSP. GF = growth factor.

(E) Cell growth of SCC9 cells in response to EGF, MSP or EGF + MSP. To allow for comparison between the separate experiments $(n=8)$, the proliferation rate of each experiment was calculated from the plot of absorbances over time as the slope of the best fit line. Then, the change in absorbances over time of the unstimulated cells was normalised to 1 and compared with those of the stimulated cells. (F) Cell growth of SCC9/shRNA-Ron cells in response to EGF, MSP or EGF + MSP. The proliferation rate and comparison among the groups were determined and performed as described $(n=3)$. The Bonferroni adjusted $P$-value is 0.17 . NS = not significant. Error bars represent standard errors in $95 \%$ confidence interval.

co-immunoprecipitated with EGFR (Figure 4D). Together, these data suggested that (1) Ron activation transphosphorylated EGFR and vice versa and (2) activated Ron biochemically interacted with EGFR.

\section{DISCUSSION}

HNSCC is the sixth most common neoplasm in the world. One obstacle to improving cure rate is the advanced stage at diagnosis and the high recurrence rate. Despite the promising result with EGFR targeting therapy in this disease, there is room for improvement. Therapies that have the potential to complement EGFR inhibitors are worth further investigations. In this study, we found that a high percentage $(64.3 \%)$ of HNSCCs expressed the Ron tyrosine kinase receptor. Unlike their premalignant counterparts, HNSCCs expressed predominantly the functional form of Ron. Although there were previous reports that showed Ron expression in over $50 \%$ of primary HNSCCs (Lin et al, 2004; Yoon et al, 2012), the sample sizes were small in these studies ( $n=8$ and 28, respectively); in addition, the methodology of detection did not have a high specificity as the IPW method and is unable to distinguish between the functional and the precursor 

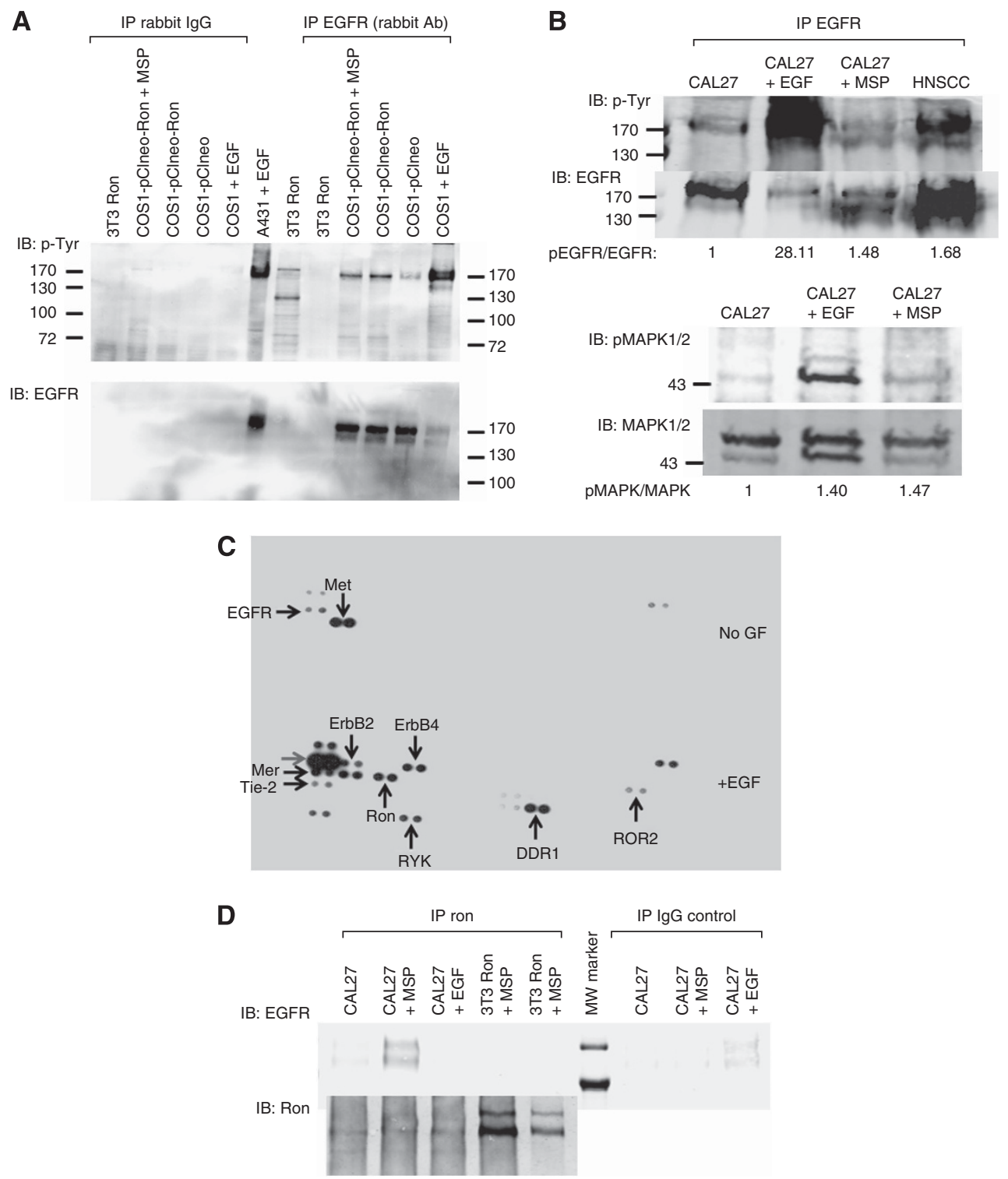

Figure 4. Biochemical analyses of Ron + HNSCC cells. (A) The EGFR transactivation by Ron overexpression in COS1 cells. The EGFR was tyrosine phosphorylated at higher levels when Ron was overexpressed (COS1-pClneo-Ron) than EGFR from mock-transfected cells (COS1-pClneo), although at lower levels than activated endogenous EGFR (COS1 + EGF) (right-most four lanes). Control precipitations with rabbit IgG were negative. The A431 + EGF cell lysates were obtained from Millipore and served as positive control. Immunoprecipitate from 3T3Ron cell lysates were negative control to ensure that Ron was not pulled down by the EGFR antibody and that the EGFR antibody used in immunoblot was specific for EGFR. (B) The EGFR transactivation by stimulating Ron in CAL27 cells. Upper panel: IPW analysis of CAL27 cells post EGF or MSP stimulation. Head and neck squamous cell carcinoma was a tumour lysate known to express high level of pEGFR and used as a positive control. The pEGFR/EGFR ratio was calculated by measuring the amount of fluorescence via a rectangle drawn around the bands using the Odyssey Infrared Imaging Program. Lower panel: MAPK signalling in CAL27 cells post EGF or MSP stimulation. The pMAPK/MAPK ratio was calculated in a similar manner as pEGFR/EGFR ratio. (C) PhosphoRTK array analysis of CAL27 cells before and after EGF stimulation. Note the baseline activation of Met in these cells (upper blot). Post EGF stimulation, EGFR became highly phosphorylated as expected (red arrow, bottom blot). GF = growth factor. (D) Co-immunoprecipitation of EGFR by Ron antibody in CAL27 + MSP cell lysate. No EGFR was detected in the other cell lysates.

The 3T3Ron + MSP cell lysates were negative controls, as 3 T3 cells do not express EGFR. Control precipitations with lgG were negative except for the trivial amount of nonspecific EGFR pulldown in the CAL27 + EGF cell lysate (upper blot). The blot was re-probed with Ron C20 antibody to confirm that the immunoprecipitation was successful (bottom blot). $\mathrm{MW}=$ molecular weight.

form of Ron. Thus, this is the largest translational study to examine Ron as a biomarker in HNSCCs.

We found that Ron + HNSCCs were more likely to locate in the oropharynx and strongly associated with pEGFR + tumours. The expression of Ron in combination with pEGFR seem to confer a lower EFS for HNSCC patients. This synergism between Ron and EGFR was also observed in both biological and biochemical assays using HNSCC cell lines. Ron expression and activation complemented EGFR effects on cell migration and growth. In addition, Ron activation augmented EGFR signalling. Although the exact mechanism of how Ron transactivated EGFR is not clear at this time, Ron/EGFR interaction has been demonstrated in other cell systems. For instance, Ron expression could functionally interact with EGF signalling in MCF10A breast cancer cells to affect cell migration and cell death. These activities of Ron did not require MSP activation or high level of Ron expression; rather, they 
were dependent on Src-family kinases (Feres et al, 2009). In addition, inhibiting EGFR signalling resulted in reduced scattering response and focus formation in cells overexpressing Ron (Peace et al, 2003); suppressing Ron expression resulted in decreased EGFR phosphorylation in J82 bladder cancer cell line (Hsu et al, 2006) and, more recently, Ron was shown to complex with EGFR to act as a transcriptional regulator in response to stress imposed on bladder cancer cells (Liu et al, 2010).

The Ron/EGFR interaction is potentially significant and might have treatment implications for HNSCCs. As EGFR inhibitors are becoming the standard therapy for HNSCCs, a complementary partner receptor like Ron might render HNSCCs less sensitive to EGFR inhibitors. In fact, several recent studies suggested that Ron expression confers resistance to not only traditional chemotherapy like cisplatin (Prislei et al, 2010) or tamoxifen (McClaine et al, 2010), but also to a targeted agent like IGF1 receptor inhibitor (Potratz et al, 2010). Given the findings in this study, it will be important to determine if targeting Ron augments the effect of EGFR inhibitors in HNSCCs. There is early evidence in a previous study that combination therapy using a neutralising Ron antibody IMC-41A10 and Erbitux led to complete tumour regression in a pancreatic cancer xenograft model (O'Toole et al, 2006), whereas Erbitux or IMC-41A10 alone only gave a partial response.

Surprisingly, recurrent HNSCCs had a dismal prognosis irrespective of whether the tumours expressed Ron/pEGFR or not (Figure 2G). In addition, these recurrent tumours were not more likely to express either receptor. This implies that Ron/EGFR might not play a strong biological role in recurrent HNSCCs. This might explain why the phase II response rates for EGFR inhibitors in recurrent HNSCCs were so low at only 5-10\% (Cohen et al, 2003; Soulieres et al, 2004; Baselga et al, 2005). These results also raise concerns as to whether the efficacy of Ron inhibitors should be tested first in recurrent HNSCCs as in traditional phase II design.

The results of this study pointed to Ron as a potential new target in HNSCCs. Although the differences in EFS between the Ron - pEGFR - , Ron + pEGFR - , Ron - pEGFR + and Ron + pEGFR + tumour groups were not statistically significant, the separation of the Kaplan-Meier curves after 2 years suggested a trend to a better EFS of the Ron-pEGFR - HNSCCs than the rest of the groups (Figure 2H). Overall, the data presented support further validation of Ron as a biomarker and therapeutic target in HNSCCs.

\section{ACKNOWLEDGEMENTS}

Tissue samples were provided by the Cooperative Human Tissue Network that is funded by the National Cancer Institute. We would like to especially thank Mrs Patti Kelly for her effort in raising the Sunrise Fund to support this work in memory and honor of her loving daughter, Lizzie Kelly. This work was supported in part by research grants from the American Cancer Society 117718MRSG-09-172-01-CCE (to ELC), the National Cancer Institute R21CA133642-01A2 (to MJH), the Stony Brook University Medical Center GCRC M01 RR10710, the Long Island League to Abolish Cancer (to ELC and MJH) and the Sunrise Fund (to ELC).

\section{REFERENCES}

Agazie YM, Hayman MJ (2003a) Development of an efficient 'substratetrapping' mutant of Src homology phosphotyrosine phosphatase 2 and identification of the epidermal growth factor receptor, Gab1, and three other proteins as target substrates. J Biol Chem 278: 13952-13958.

Agazie YM, Hayman MJ (2003b) Molecular mechanism for a role of SHP2 in epidermal growth factor receptor signaling. Mol Cell Biol 23: 7875-7886.
Ang KK, Berkey BA, Tu X, Zhang HZ, Katz R, Hammond EH, Fu KK, Milas L (2002) Impact of epidermal growth factor receptor expression on survival and pattern of relapse in patients with advanced head and neck carcinoma. Cancer Res 62: 7350-7356.

Baselga J, Trigo JM, Bourhis J, Tortochaux J, Cortes-Funes H, Hitt R, Gascon P, Amellal N, Harstrick A, Eckardt A (2005) Phase II multicenter study of the antiepidermal growth factor receptor monoclonal antibody cetuximab in combination with platinum-based chemotherapy in patients with platinum-refractory metastatic and/or recurrent squamous cell carcinoma of the head and neck. J Clin Oncol 23: 5568-5577.

Bonner JA, Harari PM, Giralt J, Azarnia N, Shin DM, Cohen RB, Jones CU, Sur R, Raben D, Jassem J, Ove R, Kies MS, Baselga J, Youssoufian H, Amellal N, Rowinsky EK, Ang KK (2006) Radiotherapy plus cetuximab for squamous-cell carcinoma of the head and neck. N Engl J Med 354: $567-578$.

Camp ER, Liu W, Fan F, Yang A, Somcio R, Ellis LM (2005) RON, a tyrosine kinase receptor involved in tumor progression and metastasis. Ann Surg Oncol 12: 273-281.

Camp ER, Yang A, Gray MJ, Fan F, Hamilton SR, Evans DB, Hooper AT, Pereira DS, Hicklin DJ, Ellis LM (2007) Tyrosine kinase receptor RON in human pancreatic cancer: expression, function, and validation as a target. Cancer 109: 1030-1039.

Casanova ML, Larcher F, Casanova B, Murillas R, Fernandez-Acenero MJ, Villanueva C, Martinez-Palacio J, Ullrich A, Conti CJ, Jorcano JL (2002) A critical role for ras-mediated, epidermal growth factor receptor-dependent angiogenesis in mouse skin carcinogenesis. Cancer Res 62: 3402-3407.

Catenacci DV, Cervantes G, Yala S, Nelson EA, El-Hashani E, Kanteti R, El Dinali M, Hasina R, Bragelmann J, Seiwert T, Sanicola M, Henderson L, Grushko TA, Olopade O, Karrison T, Bang YJ, Kim WH, Tretiakova M, Vokes E, Frank DA, Kinder HL, Huet H, Salgia R (2011) RON (MST1R) is a novel prognostic marker and therapeutic target for gastroesophageal adenocarcinoma. Cancer Biol Ther 12: 9-46.

Chan EL, Peace BE, Collins MH, Toney-Earley K, Waltz SE (2005) Ron tyrosine kinase receptor regulates papilloma growth and malignant conversion in a murine model of skin carcinogenesis. Oncogene 24: $479-488$.

Chen Q, Seol DW, Carr B, Zarnegar R (1997) Co-expression and regulation of Met and Ron proto-oncogenes in human hepatocellular carcinoma tissues and cell lines. Hepatology 26: 59-66.

Chen YQ, Zhou YQ, Fu LH, Wang D, Wang MH (2002) Multiple pulmonary adenomas in the lung of transgenic mice overexpressing the RON receptor tyrosine kinase. Recepteur d'origine nantais. Carcinogenesis 23: 1811-1819.

Cheng HL, Liu HS, Lin YJ, Chen HH, Hsu PY, Chang TY, Ho CL, Tzai TS, Chow NH (2005) Co-expression of RON and MET is a prognostic indicator for patients with transitional-cell carcinoma of the bladder. Br J Cancer 92: 1906-1914.

Cohen EE, Rosen F, Stadler WM, Recant W, Stenson K, Huo D, Vokes EE (2003) Phase II trial of ZD1839 in recurrent or metastatic squamous cell carcinoma of the head and neck. J Clin Oncol 21: 1980-1987.

DiGiovanni J, Rho O, Xian W, Beltran L (1994) Role of the epidermal growth factor receptor and transforming growth factor alpha in mouse skin carcinogenesis. Prog Clin Biol Res 387: 113-138.

Dominey AM, Wang XJ, King Jr. LE, Nanney LB, Gagne TA, Sellheyer K, Bundman DS, Longley MA, Rothnagel JA, Greenhalgh DA, Roop DR (1993) Targeted overexpression of transforming growth factor alpha in the epidermis of transgenic mice elicits hyperplasia, hyperkeratosis, and spontaneous, squamous papillomas. Cell Growth Differ 4: 1071-1082.

Etienne MC, Pivot X, Formento JL, Bensadoun RJ, Formento P, Dassonville O, Francoual M, Poissonnet G, Fontana X, Schneider M, Demard F, Milano G (1999) A multifactorial approach including tumoural epidermal growth factor receptor, p53, thymidylate synthase and dihydropyrimidine dehydrogenase to predict treatment outcome in head and neck cancer patients receiving 5-fluorouracil. Br J Cancer 79: 1864-1869.

Feres KJ, Ischenko I, Hayman MJ (2009) The RON receptor tyrosine kinase promotes MSP-independent cell spreading and survival in breast epithelial cells. Oncogene 28: 279-288.

Ford AC, Grandis JR (2003) Targeting epidermal growth factor receptor in head and neck cancer. Head Neck 25: 67-73.

Gaudino G, Follenzi A, Naldini L, Collesi C, Santoro M, Gallo KA, Godowski PJ, Comoglio PM (1994) RON is a heterodimeric tyrosine kinase receptor activated by the HGF homologue MSP. EMBO J 13: 3524-3532. 
Han S, Stuart LA, Degen SJ (1991) Characterization of the DNF15S2 locus on human chromosome 3: identification of a gene coding for four kringle domains with homology to hepatocyte growth factor. Biochemistry $\mathbf{3 0}$ : 9768-9780.

Hayman MJ, Kitchener G, Knight J, McMahon J, Watson R, Beug H (1986) Analysis of the autophosphorylation activity of transformation defective mutants of avian erythroblastosis virus. Virology 150: 270-275.

Hsu PY, Liu HS, Cheng HL, Tzai TS, Guo HR, Ho CL, Chow NH (2006) Collaboration of RON and epidermal growth factor receptor in human bladder carcinogenesis. J Urol 176: 2262-2267.

Ischenko I, Petrenko O, Gu H, Hayman MJ (2003) Scaffolding protein Gab2 mediates fibroblast transformation by the SEA tyrosine kinase. Oncogene 22: 6311-6318.

Keller J, Shroyer KR, Batajoo SK, Zhao HL, Dong LM, Hayman MJ, Chan EL (2010) Combination of phosphorylated and truncated EGFR correlates with higher tumor and nodal stage in head and neck cancer. Cancer Invest 28: 1054-1062.

Lee WY, Chen HH, Chow NH, Su WC, Lin PW, Guo HR (2005) Prognostic significance of co-expression of RON and MET receptors in node-negative breast cancer patients. Clin Cancer Res 11: 2222-2228.

Lin HS, Berry GJ, Fee Jr. WE, Terris DJ, Sun Z (2004) Identification of tyrosine kinases overexpressed in head and neck cancer. Arch Otolaryngol Head Neck Surg 130: 311-316.

Liu HS, Hsu PY, Lai MD, Chang HY, Ho CL, Cheng HL, Chen HT, Lin YJ, Wu TJ, Tzai TS, Chow NH (2010) An unusual function of RON receptor tyrosine kinase as a transcriptional regulator in cooperation with EGFR in human cancer cells. Carcinogenesis 31: 1456-1464.

Lu SL, Herrington H, Wang XJ (2006) Mouse models for human head and neck squamous cell carcinomas. Head Neck 28: 945-954.

Maggiora P, Marchio S, Stella MC, Giai M, Belfiore A, De Bortoli M, Di Renzo MF, Costantino A, Sismondi P, Comoglio PM (1998) Overexpression of the RON gene in human breast carcinoma. Oncogene 16: 2927-2933.

McClaine RJ, Marshall AM, Wagh PK, Waltz SE (2010) Ron receptor tyrosine kinase activation confers resistance to tamoxifen in breast cancer cell lines. Neoplasia 12: 650-658.

O’Toole JM, Rabenau KE, Burns K, Lu D, Mangalampalli V, Balderes P, Covino N, Bassi R, Prewett M, Gottfredsen KJ, Thobe MN, Cheng Y, Li Y, Hicklin DJ, Zhu Z, Waltz SE, Hayman MJ, Ludwig DL, Pereira DS (2006) Therapeutic implications of a human neutralizing antibody to the macrophage-stimulating protein receptor tyrosine kinase (RON), a c-MET family member. Cancer Res 66: 9162-9170.

Olayioye MA, Neve RM, Lane HA, Hynes NE (2000) The ErbB signaling network: receptor heterodimerization in development and cancer. EMBO J 19: 3159-3167.

Peace BE, Hill KJ, Degen SJ, Waltz SE (2003) Cross-talk between the receptor tyrosine kinases Ron and epidermal growth factor receptor. Exp Cell Res 289: $317-325$.

Peace BE, Hughes MJ, Degen SJ, Waltz SE (2001) Point mutations and overexpression of Ron induce transformation, tumor formation, and metastasis. Oncogene 20: 6142-6151.

Peace BE, Toney-Earley K, Collins MH, Waltz SE (2005) Ron receptor signaling augments mammary tumor formation and metastasis in a murine model of breast cancer. Cancer Res 65: 1285-1293.

Potratz JC, Saunders DN, Wai DH, Ng TL, McKinney SE, Carboni JM, Gottardis MM, Triche TJ, Jurgens H, Pollak MN, Aparicio SA, Sorensen PH (2010) Synthetic lethality screens reveal RPS6 and MST1R as modifiers of insulin-like growth factor-1 receptor inhibitor activity in childhood sarcomas. Cancer Res 70: 8770-8781.

Prislei S, Mariani M, Raspaglio G, Mozzetti S, Filippetti F, Ferrandina G, Scambia G, Ferlini C (2010) RON and cisplatin resistance in ovarian cancer cell lines. Oncol Res 19: 13-22.

Quon H, Liu FF, Cummings BJ (2001) Potential molecular prognostic markers in head and neck squamous cell carcinomas. Head Neck 23: 147-159.

Ronsin C, Muscatelli F, Mattei MG, Breathnach R (1993) A novel putative receptor protein tyrosine kinase of the met family. Oncogene 8: 1195-1202.

Rubin Grandis J, Melhem MF, Gooding WE, Day R, Holst VA, Wagener MM, Drenning SD, Tweardy DJ (1998) Levels of TGF-alpha and EGFR protein in head and neck squamous cell carcinoma and patient survival. J Natl Cancer Inst 90: 824-832.

Sibilia M, Fleischmann A, Behrens A, Stingl L, Carroll J, Watt FM, Schlessinger J, Wagner EF (2000) The EGF receptor provides an essential survival signal for SOS-dependent skin tumor development. Cell 102: 211-220.

Soulieres D, Senzer NN, Vokes EE, Hidalgo M, Agarwala SS, Siu LL (2004) Multicenter phase II study of erlotinib, an oral epidermal growth factor receptor tyrosine kinase inhibitor, in patients with recurrent or metastatic squamous cell cancer of the head and neck. J Clin Oncol 22: 77-85.

Wang MH, Iwama A, Skeel A, Suda T, Leonard EJ (1995) The murine stk gene product, a transmembrane protein tyrosine kinase, is a receptor for macrophage-stimulating protein. Proc Natl Acad Sci USA 92: 3933-3937.

Welm AL, Sneddon JB, Taylor C, Nuyten DS, van de Vijver MJ, Hasegawa BH, Bishop JM (2007) The macrophage-stimulating protein pathway promotes metastasis in a mouse model for breast cancer and predicts poor prognosis in humans. Proc Natl Acad Sci USA 104: $7570-7575$.

Willett CG, Wang MH, Emanuel RL, Graham SA, Smith DI, Shridhar V, Sugarbaker DJ, Sunday ME (1998) Macrophage-stimulating protein and its receptor in non-small-cell lung tumors: induction of receptor tyrosine phosphorylation and cell migration. Am J Respir Cell Mol Biol 18: 489-496.

Woodworth CD, Gaiotti D, Michael E, Hansen L, Nees M (2000) Targeted disruption of the epidermal growth factor receptor inhibits development of papillomas and carcinomas from human papillomavirus-immortalized keratinocytes. Cancer Res 60: 4397-4402.

Yoon TM, Kim SA, Park YL, Lee KH, Sung MW, Lee JK, Lim SC, Chung IJ, Joo YE (2012) Expression of the receptor tyrosine kinase recepteur d'origine nantais and its association with tumor progression in hypopharyngeal cancer. Head Neck; e-pub ahead of print 6 August 2012; doi:10.1002/hed.23090.

Zhao S, Ammanamanchi S, Brattain M, Cao L, Thangasamy A, Wang J, Freeman JW (2008) Smad4-dependent TGF-beta signaling suppresses RON receptor tyrosine kinase-dependent motility and invasion of pancreatic cancer cells. J Biol Chem 283: 11293-11301.

Zinser GM, Leonis MA, Toney K, Pathrose P, Thobe M, Kader SA, Peace BE, Beauman SR, Collins MH, Waltz SE (2006) Mammary-specific Ron receptor overexpression induces highly metastatic mammary tumors associated with beta-catenin activation. Cancer Res 66: 11967-11974.

This work is published under the standard license to publish agreement. After 12 months the work will become freely available and the license terms will switch to a Creative Commons AttributionNonCommercial-Share Alike 3.0 Unported License.

Supplementary Information accompanies this paper on British Journal of Cancer website (http://www.nature.com/bjc) 